\title{
An Integrated Knowledge Translation Study to Address Avoidable Rehospitalisations and Unplanned Admissions for Older People: Study Protocol
}

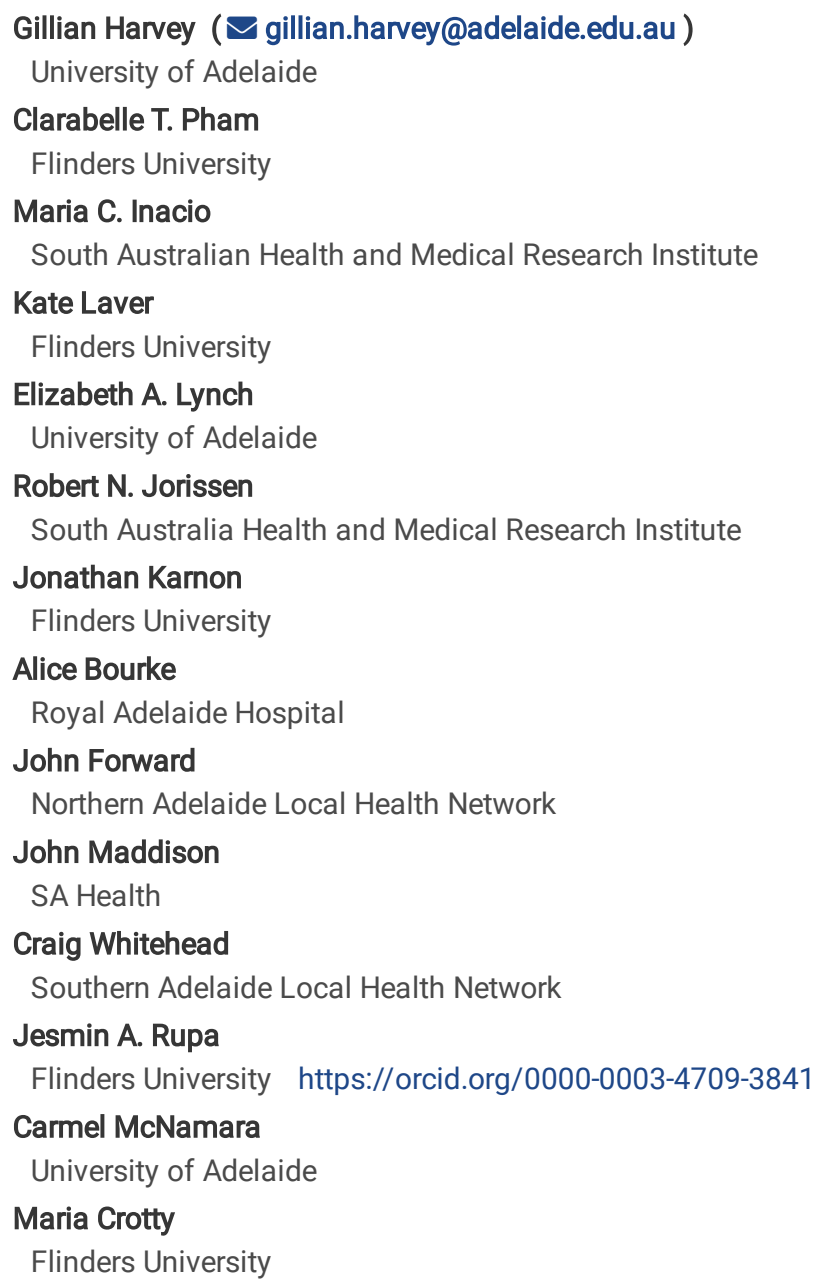

\section{Study Protocol}

Keywords: Hospitalisations, Unplanned rehospitalisations, Emergency department encounters, Older people, Evaluation, Integrated knowledge translation, Quality improvement

Posted Date: October 21 st, 2020

DOI: https://doi.org/10.21203/rs.3.rs-93290/v1

License: (c) (1) This work is licensed under a Creative Commons Attribution 4.0 International License. Read Full License 


\section{Abstract}

\section{Background}

Repeated admission to hospital can be a stressful and negative experience for older people and their families and puts additional pressure on the health care system. While there is evidence about strategies to better integrate care and improve older patients' experiences at transitions of care, thus helping to reduce hospital readmissions, implementing these strategies at scale is challenging. The objective of this project is to improve the pathways of care for older people after discharge from hospital by leveraging existing large datasets and an established collaborative network of clinicians, consumers, academics, and aged care providers.

\section{Methods}

The project will be undertaken in the state of South Australia and focuses on people aged 65 and over. A mixed methods approach will be adopted, with three inter-linked work packages that aim to: analyse existing registry data to profile individuals at high risk of emergency department encounters and hospital admissions; evaluate the effectiveness and cost-effectiveness of existing 'out-of-hospital' programs provided within the state; implement and evaluate a statewide quality improvement collaborative to tackle key interventions likely to improve older people's care at points of transitions. The study is underpinned by an integrated approach to knowledge translation, with active engagement of a broad range of stakeholders throughout, to optimize the relevance and sustainability of the changes that are introduced.

Discussion

This project highlights the uniqueness and potential value of a multi-faceted approach (risk profiling; evaluation framework; implementation and evaluation) to improving health services. The project aims to develop a practical and scalable solution to a challenging health service problem for frail older people and service providers.

Trial registration

Not applicable.

\section{Background}

Avoidable hospitalisations of older people remain a global challenge for health-care systems (1-3). Additionally, the number of unplanned visits to emergency departments (ED) has continued to grow even though many countries have achieved substantial health care improvements in recent decades (4-6). Increased rate of hospitalisations is burdensome for health care systems and undesirable for patients, particularly for older people with multiple co morbidities (7).

Existing literature identifying the risk factors associated with hospitalisations indicates that there are no simple solutions. Studies have identified socio-demographic and environmental factors such as higher age, being male, level of educational attainment, marital status, geographical location, socio-economic deprivation, living alone without help, reduced social activity and community engagement as potential risk factors for hospitalisations and ED presentations (2,8-16). Additionally, a wide range of medical conditions and health care related factors such as self-rated poor health status, frailty, frequent falls, co-morbidity, polypharmacy, overuse and underuse of medications, depression, anxiety, heart failure, cognitive impairment, higher number of primary care visits and admission to nursing home have also been identified (5, 17-22). However, few studies have examined the combination of individual, medication, system, and hospital-associated factors that together could be associated with frequent hospital admissions.

A previous history of hospitalisations is associated with a higher risk of avoidable rehospitalisations, particularly in older people aged 65 and above $(11,23)$. An avoidable rehospitalisation occurs when a patient who has been discharged from hospital is admitted again within a certain time interval, where the readmission could potentially have been avoided through improved clinical management or appropriate discharge planning $(23,24)$. Unplanned hospital readmissions are important to patients, families, clinicians, and policy makers since reducing their occurrence can improve care and reduce the costs of health care (8). However, the evidence of policy interventions that effectively reduce unplanned hospital readmissions in older people is sparse.

The importance of collaboration between the spectrum of care providers such as hospital, aged care, local agencies and primary care (25-27) is highlighted in the literature to support interventions aimed at reducing unplanned rehospitalisations. Moreover, patient education, home follow-up, home monitoring, adjustment of medication and regular communication with clinical experts have been found to be effective in reducing unplanned rehospitalisations in older people (28). A recent systematic review suggested that self-management, telephone follow-up and medication reconciliation activities are most likely to be effective in reducing hospital readmission (29).

Similar to other international experiences reported in the literature, in Australia, older people with multiple long-term conditions have complex health and social care needs, which increases their risk of repeated hospitalisations. Australians aged 65 years and over represent $15 \%$ of the population 
yet account for $20 \%$ of ED presentations and $42 \%$ of same day hospitalisations; people aged 85 years and over account for $23 \%$ of all the presentations for people aged 65 years and over (30). Many hospital admissions occur for ambulatory care sensitive conditions where hospital admission could have been prevented by interventions in primary care $(31,32)$.

Older people aged 65 and over made up 18\% of South Australia's (SA) population, which is higher than the national average (30). Current approaches to addressing the problem of avoidable hospital admissions and readmissions in SA differ between various administrative structures within the state. For example, some health services for the older population are only available within particular local health networks (LHNs), and evaluation criteria between services differ. Therefore, it is unclear which services are most appropriate and effective for which patients and, at what cost to individuals and the state health service.

The State Action on Avoidable Rehospitalisations and Unplanned Admissions across South Australia (STAAR-SA) project was designed to establish a state-wide approach, to better understand the problems and design, implement, and evaluate appropriate local solutions. The study is applying a co-design approach, engaging with different stakeholder groups throughout the study to embed an integrated approach to knowledge translation $(33,34)$. This involves a number of different activities, namely: establishing a partnership of stakeholders; understanding the issue of rehospitalisations and unplanned admissions of the older population; evaluating current out of hospital programs for older people; and translating evidence into practice through a quality improvement collaborative (QIC). Table 1 comprises the co-KT stages of the study.

Table 1: Project aims mapped against an integrated knowledge translation (KT) approach

\section{Project objectives}

1. Establish a partnership with health providers, aged care non-government agencies (NGOs), general practices, consumers \& researchers to guide the study.

2. Profile the older SA population at risk of multiple hospitalisations.

Associated stages of the integrated approaches to KT

Building the stakeholder partnerships \& collaboration required for integrated knowledge translation.

Problem identification/ clarification \& knowledge creation.

3. Develop an evaluation framework for health services to evaluate out of hospital programs for older people.

Knowledge synthesis to create an evidence 'product'.

4. Establish a quality improvement collaborative where front-line clinicians \& their service managers (working within the partnership organisations) come together to learn, share and benchmark outcomes Implementation \& evaluation. with the goal of increasing the quality of care for older people at times of transition.

The governance structure includes stakeholders, an expert panel of knowledge users (e.g. front-line clinicians, managers) and researchers who will work together with consumers to collaboratively define and address gaps between knowledge and practice. The benefit of the integrated KT approach is that the expertise and skills of both the researchers and knowledge users are explicitly recognised, and through collaboration, any discrepancies between the existing evidence and the needs of the health service can be identified and subsequently addressed. $(35,36)$. In this way, the KT approach is intended to generate rapid translation and enhanced impact.

\section{Methods/design Objectives}

The overarching aim of the project will be addressed through three inter-connected Work Packages (Fig. 1)

Work Package 1 (WP1) comprises three objectives to characterize individuals most at risk of hospitalisations at vulnerable periods (i.e. at time of an aged care eligibility assessment or entry into permanent residential aged care) and identify opportunities to reduce morbidity and utilisation:

1. To construct predictive models for any unplanned hospitalisation after a first-time aged care eligibility assessment and first-time entry into permanent residential aged care. To examine whether individual-related characteristics, medication-related characteristics, system-related characteristics, and hospital-related characteristics are predictive of unplanned hospitalisation.

2. To construct predictive models for emergency department encounters after a first-time aged care eligibility assessment and first-time entry into permanent residential aged care. To examine whether individual-related characteristics, medication-related characteristics, system-related characteristics, and hospital-related characteristics are predictive of emergency department encounters.

3. To construct predictive models for mortality after a first-time aged care eligibility assessment and first-time entry into permanent residential aged care. To examine whether individual-related characteristics, medication-related characteristics, system-related characteristics, and hospital-related characteristics are predictive of mortality. 
Within the cohort of WP1, there are people with multi-morbidity, complex co-morbidities, and high frailty, which are known to disproportionally affect health service utilisation and wellbeing. Importantly, greater health service utilisation is not always associated with better health outcomes. Undertaking this work will:

1. Allow the researchers and participants in the quality improvement collaborative to describe and understand the incidence of state-wide hospitalisation and emergency department encounters for individuals both at the time of first screening for aged care eligibility and entry into permanent aged care services.

2. Develop and validate risk profiling tools for hospitalisations and emergency department encounters that can be used for decision-making support, individualisation of care plans, and educational purposes for individuals at vulnerable periods in their aged care journey.

The analyses from WP1 will characterise older people that are hospitalised in three metropolitan and six regional LHNs the four state LHNs in SA. We will work with our stakeholder partnership to identify appropriate ways to present and apply the risk profiling tools.

Work Package 2 (WP2) comprises three objectives to evaluate selected existing out-of-hospital care services in South Australia:

1) To compare outcomes, from routinely collected data, up to 12 months after commencing an out-of-hospital care service, compared with the same outcomes in:

1. the two years prior to commencing the out-of-hospital care service

2. a control group (who did not commence the program) selected from the Registry of Senior Australians (ROSA), an Australian Clinical Quality Registry designed to monitor people accessing aged care services.

The main outcomes include number and duration of unplanned admissions to a hospital, number of presentations to an emergency department, number of primary care visits and mortality.

2) To assess whether the costs of delivering the out-of-hospital care service is offset by reduced health system costs (e.g. primary care utilisation, hospital admissions and emergency department presentations).

There are many services in SA that aim to improve transitions of care for older people and avoid hospitalisations. However, services report varied outcomes, and do not report on the same key indicators such as rates of hospital readmission, length of hospital stays or health care costs. An evaluation framework will be developed to provide a standardized approach for the monitoring, reporting and assessment of the costs and outcomes of out-of-hospital care services.

This framework will also include a qualitative component to explore the consumer/carer experiences of the selected out-of-hospital services to inform and provide context to the quantitative component. The predictive models from WP1 will inform the matching algorithm to identify the control group from ROSA and the potential for priority setting based on risk of hospitalisation (i.e. which services work best for whom, where and when).

Work Package 3 (WP3) will involve the establishment of a state-wide quality improvement collaborative (QIC) to improve the quality of care for older people at times of transition.

The QIC model is based on evidence that assessing one's own progress and benchmarking with other professionals can facilitate faster and wider implementation of quality improvement practices (37). We will replicate in part the STAAR improvement collaborative from the US, utilising the same four-part change model (38).

Firstly, we will convene a panel of experts (representing a variety of health and aged care providers, consumers and academics from contexts). Expert panel members will be presented with the latest evidence about hospital transitions from both quantitative and qualitative studies and then tasked with identifying the main modifiable problems which contribute to avoidable hospital readmission in South Australia and suggest specific strategies to manage these. The panel will also be asked to identify outcomes or process indicators that can be used to monitor change over time.

Previous syntheses of the published literature have identified several key processes that are likely to be associated with effective transitions for older people (38) and these will be considered in the panel deliberations, for example:

- Use of the same medical record across sectors

- Formal collaboration with families to develop and share care plans

- Actions taken to plan for potential problems (e.g. General Practitioner appointments within 30 days of discharge, single point of contact for relevant services)

- Follow up via a phone call within 48 hours of discharge. 
Once the key modifiable problems, process indicators and possible strategies have been established, we will advertise amongst our networks and health and aged care services in South Australia to identify and recruit teams to participate in the QIC. Our QIC will work to achieve high levels of adherence to process indicators (reflecting priority outcomes) across the array of existing services. Regardless of existing funding arrangements, the aim is for consistency in key aspects of care delivery. Members of the collaborative will (1) be offered information and education about best practice for each process indicator, (2) be supported to identify existing barriers to implementation, (3) develop quality improvement plans with the support of clinical and quality improvement experts, (4) implement strategies to increase adherence with the support of clinical and quality improvement experts, and (5) benchmark with others in the collaborative. The QIC members will participate in three learning meetings over a period of approximately nine months; the purpose of the first meeting is to establish the quality improvement targets, the purpose of the second is to reflect and discuss progress with other teams, and the purpose of the third meeting is to share outcomes and learnings. Over the course of the QIC, teams will be supported by a member of the project team in a QIC facilitator role, providing coaching, encouragement and help with problem solving and implementation, as required.

The researchers will evaluate the success of the QIC by (1) measuring changes in adherence to selected process indicators within each service, (2) assessing levels of improvement knowledge in collaborative members - pre and post involvement, (3) conducting interviews and/or focus groups to ascertain attitudes towards the collaborative and, (4) measuring new linkages between key personnel using social network analysis. We will also collect data on collaborative projects, presentations, publications, and grant applications arising from the QIC.

\section{Context and Setting}

This project includes the three metropolitan and six regional LHNs, the two state based primary health networks (PHN) that coordinate health services in local areas, three aged care providers, one consumer organisation and academics from the three largest SA universities and the South Australian Health and Medical Research Institute (SAHMRI). LHNs manage the delivery of public hospital services and other community-based health services as determined by the state government. Table 2 highlights a summary of hospital and self-reported health status of inpatients across South Australia's metropolitan and regional major public hospitals under LHNs. Prior co-designed research undertaken by project team members to explore the experiences and perspectives of older people, their families, carers and service providers in relation to managing their condition, remaining well at home and avoiding unnecessary hospitalisation, also provided important background information for the current study $(39,40)$.

Table 2: Hospital and health status of South Australia's LHNs

\begin{tabular}{|llll|}
\hline Local Health Networks (LHNs) & Total bed ${ }^{*}$ capacity & Population $^{* *}$ & Health status $^{* * *}(\%)$ \\
\hline Metro SA LHNs & & & \\
\hline Central Adelaide LHN & 1,412 & 1,280 & $84.8 \%$ \\
\hline Northern Adelaide LHN & 505 & 546 & $79.3 \%$ \\
\hline Southern Adelaide LHN & 917 & 815 & $83.0 \%$ \\
\hline Regional SA LHNs & 577 & 347 & $77.9 \%$ \\
\hline
\end{tabular}

* The total general and specialist base bed capacity for the hospitals. **The total number of patients currently occupying all hospital beds. Data source: SA Health website:

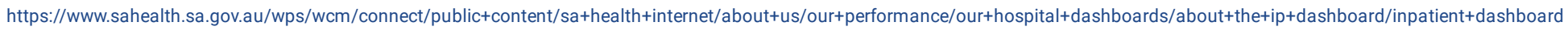
*** Self-reported health status as good, very good or excellent (aged 18+years) 2016. Data source: SALHN Clinical Services Plan $2020-2024$.

\section{Participants}

WP1 will use ROSA, which includes longitudinal information on pathways and transitions in aged care linked with participant encounters with the health care sector and mortality. ROSA has a national historical (1997-2016, 2.9 million participants) and a prospective SA based (2018-ongoing, 16,000 participants/year) cohort. WP1 will include any individuals who either completed an Aged Care Assessment Team (ACAT) assessment between January 2012-May 2016 or entered permanent residential aged care between 2013-2016 (who also have timely ACAT data).

In WP2, out-of-hospital care services aimed at managing people with chronic conditions to reduce unplanned rehospitalisations in the metropolitan and regional LHNs in South Australia will be evaluated. This will be done through the collation of data on a retrospective cohort of patients who have received a service and comparing them with a control group (similar patient characteristics but have not received the service) within ROSA. WP2 will include South Australian individuals aged 65 years and over who have had an ACAT assessment to enable linkage to ROSA. The study cohort will comprise individuals in ROSA who have received a service between April 2015 and December 2017. The historical control group will comprise individuals in ROSA who have not received a service between January 2013 and March 2015. 
For WP3, health and aged care teams will be invited to participate in the QIC through a combination of purposive and snowball sampling. Teams may be working in acute, primary care or community settings and employed by government or non-government organisations.

\section{Exposures of interest}

In each of the two cohorts of WP1, the study includes individual, medication, system, and hospital-related characteristics. Individual-related characteristics include age, gender, marital status, health conditions, veteran and concession card status, cognitive impairment, depression status, activities of daily living limitations, behavioural and complex health care level. Medication-related characteristics include polypharmacy, dispensing of medications known to be associated with hospitalisations and any potentially inappropriate medications in older people. Facility/provider characteristics, geographical area, state, and year are included as system related characteristics. Finally, history of hospitalisations and emergency department encounters, history of primary care and specialist encounters, time between previous hospital discharge and ACAT assessment will be examined as hospital and health care related characteristics.

For WP2, potentially eligible out-of-hospital services will be nominated by LHNs, PHNs, SA Ambulance Service and NGOs. To be eligible for the quantitative evaluation, services had to commence before December 2017, have an adequate sample size of at least 100 patients enrolled by December 2017, and have patient identifiers (full name, date of birth and Medicare number or hospital admission date) to enable linkage to the historical ROSA. Data on the retrospective patient cohorts from included out-of-hospital care services will be analysed.

\section{Outcomes of interest}

The anticipated outcomes from the Work Packages of this study are presented in Table 3.

Table 3: STAAR-SA potential project outcomes

\begin{tabular}{|c|c|}
\hline $\begin{array}{l}\text { Work } \\
\text { Packages }\end{array}$ & Outcome details \\
\hline $\begin{array}{l}\text { WP1 } \\
\text { Risk profile }\end{array}$ & $\begin{array}{l}\text { Risk profiling tools for hospitalisation. For each of the following outcomes, the time is measured from either date of } \\
\text { completion of aged care eligibility assessment (cohort 1) or date of entry into permanent residential aged care (cohort 2). Time } \\
\text { period of outcome evaluation : January 2012-May 2016. } \\
\text { - Time to unplanned hospitalisation after entry into the relevant cohort } \\
\text { - Time to ED encounter } \\
\text { - Time to death }\end{array}$ \\
\hline $\begin{array}{l}\text { WP2 } \\
\text { Evaluation } \\
\text { framework }\end{array}$ & $\begin{array}{l}\text { For each out-of-hospital care service: } \\
\text { - Cost per unplanned hospitalisation } \\
\text { - Cost per bed day saved (includes the costs for hospitalisation, emergency department presentations and primary care) } \\
\text { - Consumer/carer experience } \\
\text { - Mortality } \\
\text { - The extent to which the program reaches the intended target group } \\
\text { - Variations in implementation of the program between different population recipients }\end{array}$ \\
\hline $\begin{array}{l}\text { WP3 } \\
\text { Collaborative }\end{array}$ & $\begin{array}{l}\text { - Establishment of a network of people working in Out of Hospital Care in SA } \\
\text { - Formation of linkages across LHNs, primary care settings and non-government aged care providers } \\
\text { - Increased adherence to a core set of quality process indicators for transitions in care for older people } \\
\text { - Improved quality of care transitions } \\
\text { - Increased capability for quality improvement }\end{array}$ \\
\hline
\end{tabular}

\section{Data analysis}

The data analysis of this study will be both quantitative and qualitative in nature, based on the individual Work Package objectives.

In WP1 the cohort and crude outcomes will be described using means, standard deviation, medians, interquartile ranges (IQR), frequency and proportions. For most predictive models for hospitalisation and ED encounters, a Fine-Gray model will be employed with death as a competing risk. 
For predictive models for mortality Cox regressions will be employed. The proportional hazards assumptions will be tested using Schoenfeld residuals. Prediction models will be assessed by application to training set data (January 2013-May 2016) using 10-fold cross-validation and to external validation data (2012). Sub-distribution hazard ratios and 95\% confidence intervals will be presented for examined factors found to be predictors of the outcomes of interest. The models' calibration will be examined by comparing observed and predicted mean survival where the groups are defined over quantiles or deciles of the predictions at specific time periods. Discrimination will be examined using Harrell's C-index.

In WP2, interrupted time series (ITS) analysis will be performed to explore the impact of each out-of-hospital service on unplanned hospital admissions. This will involve tracking a period before and after the commencement of each service and assess any changes in the mean unplanned hospital admissions over time. To compare the costs and outcomes in patients who did (intervention) and did not (control) receive the out-of-hospital service, coarsened exact matching (CEM) and then a comparison of the differences in the mean costs and outcomes on the matched data will be performed. CEM temporarily coarsens the intervention patient demographic data (e.g. using coarse age groups rather than exact birthdays) and then finds control patients that are exact matches to the intervention patients. For each CEM application, standardised differences in the mean values will be estimated to assess balance in the variables used to match the intervention and control groups. Each intervention patient will be matched with up to 3 control patients. The 1:3 matching ratio will maximise the statistical power to detect a difference in outcomes between the intervention and control.

Data analysis in WP3 will involve both quantitative and qualitative methods. Changes in adherence to selected process indicators within each service and pre-post levels of knowledge in collaborative members will be analysed using descriptive statistics. Qualitative interview and focus group data will be subject to inductive, thematic analysis (41).

\section{Discussion}

Reducing rehospitalisations in older people is complex and requires sophisticated knowledge translation strategies (42, 43). Accordingly, our protocol incorporates engagement with a myriad of different stakeholder groups and allows for iterations and contextualisation throughout the process. Applying a novel and innovative approach, our aim is to address a complex problem at a state health system level in a way that recognises and accommodates differences in resources, knowledge, skills and culture. Once established, the network will provide a platform of knowledge users, researchers and policy makers that has the potential to tackle other complex problems associated with caring for the older population across SA.

\section{Abbreviations}

South Australia: SA; ROSA: Registry of Senior Australians; NGO: Non-Government Organization; IKT: Integrated Knowledge Translation; ED: Emergency Department; QIC: Quality Improvement Collaborative; LHN: Local Health Network; ACAT: Aged Care Assessment Team; SAHMRI: South Australian Health and Medical Research Institute; AlHW: Australian Institute of Health and Welfare; IQR: Interquartile ranges; ITS: Interrupted Time Series; CEM: Coarsened Exact Matching.

\section{Declarations}

\section{Ethics approval and consent to participate}

The study got ethics approval for WP1from SA Department for Health and Wellbeing Human Research Ethics Committee (Ref: HREC/18/SAH/90), Australian Institute of Health and Welfare (Ref: E02018/1/418) and University of South Australia (UniSA ID: 200489). The SA Department for Health and Wellbeing Human Research Ethics Committee also approved the ethics for WP 2 (Ref: HREC/20/SAH/32) and WP 3 (Ref: HREC/20/SAH/46/AM01. For WP1 and WP2, we got approval to waive the requirement for consent for the patients in the datasets for analysis (WP1 and WP2) since the risk to patients with involvement would be considered no more than low risk. Moreover, the data provided to the research team will be in a de-identified format and we are using secured and approved servers for the storage and analysis. For WP3, participants will be provided a Participant Information Sheet including an overview of the study and what the requirements are should they consent to participate. A consent and withdrawal form will also be provided. The participants will be asked again before the interview commences, to ensure they are comfortable and consent to participate.

\section{Consent for publication}

Not applicable.

\section{Availability of data and materials}

No data and materials from any individual person will be published, thus consent for publication will not be applicable. 


\section{Competing interests}

The authors declare that they have no competing interests.

\section{Funding}

The STAAR-SA program is funded by a Medical Research Future Fund Rapid Applied Research Translation Grant on behalf of Health Translation South Australia (HTSA).

\section{Authors' contributions}

Maria Crotty and Craig Whitehead conceived of the study and reviewed the study protocol. Gillian Harvey wrote and edited the paper. Maria Inacio and Robert Jorissen are the leads for WP1; Clarabelle Pham and Jonathan Karnon are the leads for WP2, and Elizabeth Lynch and Kate Laver are the leads for WP3. All Work package leads wrote and reviewed the study protocol. Other co-investigators Alice Bourke, John Maddison, Jon Forward, and Carmel McNamara also reviewed the protocol study paper. Jesmin Rupa wrote and assisted in drafting and editing the study. All authors read and approved the final protocol paper.

\section{Acknowledgements}

We would like to thank SAHMRI, AIHW, SA Health and the entire STAAR-SA stakeholder group for supporting this study. We further would like to thank the ROSA research team for providing the data for WP1 and WP2. The ROSA director, Associate Professor Maria Inacio is supported by The Hospital Research Foundation Mid-Career Fellowship (MCF-27-2019).

\section{References}

1. Busby J, Purdy S, Hollingworth W. A systematic review of the magnitude and cause of geographic variation in unplanned hospital admission rates and length of stay for ambulatory care sensitive conditions. BMC Health Serv Res. 2015; doi: 10.1186/s12913-015-0964-3:15, 324.

2. Dahlberg L, Agahi N, Schön P, Lennartsson C. Planned and unplanned hospital admissions and their relationship with social factors: Findings from a national, prospective study of people aged 76 years or older. Health Serv Res. 2018. 53: 4248-4267.

3. Visade F, BabykinaG, Lamer A, Defebvre MM, Verloop D, Ficheur G, Genin M, Puisieux F, Beuscart JB. Importance of previous hospital stays on the risk of hospital re-admission in older adults: a real-life analysis of the PAERPA study population. Age Ageing. 2020; doi:10.1093/ageing/afaa139.

4. Deeny S, Thorlby R, Steventon A. Briefing: Reducing emergency admissions: unlocking the potential of people to better manage their long-term conditions. The Health Foundation. 2018. https://www.health.org.uk/publications/reducing-emergency-admissions-unlocking-the-potential-ofpeople-to-better-manage-their-long-term-conditions. Accessed 3 Sept 2020.

5. Morris JN, Howard EP, Steel K, Schreiber R, Fries, BE, Lipsitz LA, Goldman B. Predicting risk of hospital and emergency department use for home care elderly persons through a secondary analysis of cross-national data. BMC Health Serv Res. 2014; 14 : 1-11.

6. Steventon, A, Deeny S, Friebel R, Gardner T, Thorlby R. Emergency hospital admissions in England. The Health Foundation. 2018. www.health.org.uk/publication/emergency-hospital-admissions-england-which-may-be-avoidable-and-how. Accessed 3 Sept 2020.

7. Facchinetti G, D’Angelo D, Piredda M, Petitti T, Matarese M, Oliveti A, De Marinis MG. Continuity of care interventions for preventing hospital readmission of older people with chronic diseases: A meta-analysis. Int J Nurs Stud. 2020; doi: 10.1016/j.ijnurstu.2019.103396.

8. Berry JG, Gay JC, Joynt Maddox K, Coleman EA, Bucholz EM, O’Neill MR, Blaine K, Hall M. Age trends in 30-day hospital readmissions: US national retrospective analysis. BMJ 360, 2018; doi: 10.1136/bmj.k497.

9. Donnan PT, Dorward DW, Mutch B, Morris AD. Development and validation of model for predicting emergency admissions over the next year (PEONY): a UK historical cohort study. Arch Intern Med. 2008; 168: 1416-1422.

10. Inouye SK, Zhang Y, Jones RN, Shi P, Cupples LA, Calderon HN, Marcantonio ER. Risk factors for hospitalization among community-dwelling primary care older patients: development and validation of a predictive model. Med Care. 2008; 46: 726-731.

11. Landi F, Onder G, Cesari M, Barillaro C, Lattanzio F, Carbonin PU, Bernabei R. Comorbidity and social factors predicted hospitalization in frail elderly patients. J Clin Epidemiol. 2004; 57:832-836.

12. Lopez-Aguila S, Contel JC, Farre J, Campuzano JL, Rajmil L. Predictive model for emergency hospital admission and 6-month readmission. Am J Manag Care. 2011; 17: e348-e357.

13. Longman JM, Rolfe MI, Passey MD, Heathcote KE, Ewald DP, Dunn T, Barclay L, Morgan GG. Frequent hospital admission of older people with chronic disease: a cross-sectional survey with telephone follow-up and data linkage. BMC Health Serv Res. 2012; doi: 10.1186/1472-6963-12-

Page $8 / 10$ 
373.

14. Roos LL, Walld R, Uhanova J, Bond. Physician visits, hospitalizations, and socioeconomic status: ambulatory care sensitive conditions in a Canadian setting. Health Serv Res. 2005; 40:1167-1185.

15. Shepherd H, Livingston G, Chan J, Sommerlad A. Hospitalisation rates and predictors in people with dementia: a systematic review and metaanalysis. BMC Med. 2019; doi: 10.1186/s12916-019-1369-7.

16. Wallace E, Stuart E, Vaughan N, Bennett K, Fahey T, Smith SM. Risk prediction models to predict emergency hospital admission in communitydwelling adults: a systematic review. Med Care. 2014; 52: 751-765.

17. Albert SM, Colombi A, Hanlon J. Potentially inappropriate medications and risk of hospitalization in retirees. Drugs Aging, 2010; $27: 407-415$.

18. Barreto Pde S, Greig C, Ferrandez AM. Detecting and categorizing frailty status in older adults using a self-report screening instrument. Arch Gerontol Geriatr, 2012; 54: e249-e254.

19. Fogg C, Griffiths P, Meredith P, Bridges J. Hospital outcomes of older people with cognitive impairment: An integrative review. Int J Geriatr Psychiatry. 2018; 33:1177-1197.

20. Boockvar K, Lachs M. Hospitalization risk following admission to an academic nursing home. J Am Med Dir Assoc. 2002; 3:130-135.

21. Daly MR, Mellor JM, Millones M. Do avoidable hospitalization rates among older adults differ by geographic access to primary care physicians? Health Serv Res. 2018; 53 Suppl 1:3245-3264.

22. Rosano A, Loha CA, Falvo R, van der Zee J, Ricciardi W, Guasticchi G, de Belvis AG. The relationship between avoidable hospitalization and accessibility to primary care: a systematic review. Eur J Public Health. 2013; 23:356-360.

23. Considine J, Fox K, Plunkett D, Mecner M, O'Reilly M, Darzins P. Factors associated with unplanned readmissions in a major Australian health service. Aust Health Rev. 2019; 43:1-9.

24. Kristensen SR, Bech M, Quentin W. A roadmap for comparing readmission policies with application to Denmark, England, Germany and the United States. Health Policy. 2015; 119:264-73.

25. Hansen LO, Young RS, Hinami K, Leung A, Williams MV. Interventions to reduce 30-day rehospitalization: a systematic review. Ann Intern Med. 2011; 155:520-528.

26. McCarthy D, Ryan J, Klein S. Models of care for high-need, high-cost patients: an evidence synthesis. 1st ed. New York (NY): Commonwealth Fund; 2015.

27. Philp I, Mills KA, Thanvi B, Ghosh K, Long, JF. Reducing hospital bed use by frail older people: results from a systematic review of the literature. Int J Integr Care. 2013; doi: 10.5334/ijic.1148.

28. Linertová R, García-Pérez L, Vázquez-Díaz JR, Lorenzo-Riera A, Sarría-Santamera A. Interventions to reduce hospital readmissions in the elderly: in-hospital or home care. A systematic review. J Eval Clin Pract. 2011; 17:1167-1175.

29. Tomlinson J, Cheong VL, Fylan B, Silcock J, Smith H, Karban K, Blenkinsopp A. Successful care transitions for older people: a systematic review and meta-analysis of the effects of interventions that support medication continuity. Age Ageing. 2020; doi:10.1093/ageing/afaa002.

30. Australian Institute of Health and Welfare (AlHW). 2018, Older Australia at a glance. 2018.https://www.aihw.gov.au/reports/older-people/olderaustralia-at-a-glance. Accessed 3 Sept 2020.

31. Banham D, Karnon J, Densley K, Lynch JW. How much emergency department use by vulnerable populations is potentially preventable?: A period prevalence study of linked public hospital data in South Australia. BMJ Open. 2019; doi: 10.1136/bmjopen-2018-022845.

32. Falster M, Jorm L. A guide to the potentially preventable hospitalisations indicator in Australia. Centre for Big Data Research in Health. University of New South Wales in consultation with Australian Commission on Safety and Quality in Health Care and Australian Institute of Health and Welfare. 2017.

33. Kitson A, Powell K, Hoon E, Newbury J, Wilson A, Beilby J. Knowledge translation within a population health study: how do you do it?. Implement Sci. 2013; doi:10.1186/1748-5908-8-54.

34. Graham ID, Kothari A, McCutcheon C. Moving knowledge into action for more effective practice, programmes and policy: protocol for a research programme on integrated knowledge translation. Implement Sci. 2018; doi: 10.1186/s13012-017-0700-y.

35. Kothari A, Wathen CN. A critical second look at integrated knowledge translation. Health Policy. 2013; 109:187-191.

36. Salsberg J. Integrated knowledge translation and participatory research. In Graham ID, Tetro JM, Pearson A, editors. Turning knowledge into action: practical guidance on how to do integrated knowledge translation research. Lippincott Williams \& Wilkins; 2014.

37. Shaw EK, Chase SM, Howard J, Nutting PA, Crabtree BF. More black box to explore: how quality improvement collaboratives shape practice change. J Am Board Fam Med. 2012; 25: 149-157.

38. McCarthy D, Johnson MB, Audet AM. Recasting readmissions by placing the hospital role in community context. JAMA. 2013; $309: 351-352$.

39. Dollard J, Harvey G, Dent E, Trotta L, Williams N, Beilby J, Hoon E, Kitson A, Seiboth C, Karnon J. Older people who are frequent users of acute care: A symptom of fragmented care? A case series report on patients' pathways of care. J Frailty Aging. 2018; 7:193-195. 
40. Mittinty MM, Marshall A, Harvey G. What integrated care means from an older person's perspective? A scoping review protocols. BMJ Open. 2018; doi: 10.1136/bmjopen-2017-019256.

41. Braun V, Clarke V. Using thematic analysis in psychology. Qualitative research in psychology. 2006; 3:77-101.

42. Braithwaite J, Churruca K, Long JC, Ellis LA, Herkes J. When complexity science meets implementation science: a theoretical and empirical analysis of systems change. BMC Med. 2018; doi:10.1186/s12916-018-1057-z.

43. Kitson A, Brook A, Harvey G, Jordan Z, Marshall R, O’Shea R, Wilson D. Using complexity and network concepts to inform healthcare knowledge translation. Int J Health Policy Manag. 2018; 7:231-243.

\section{Figures}

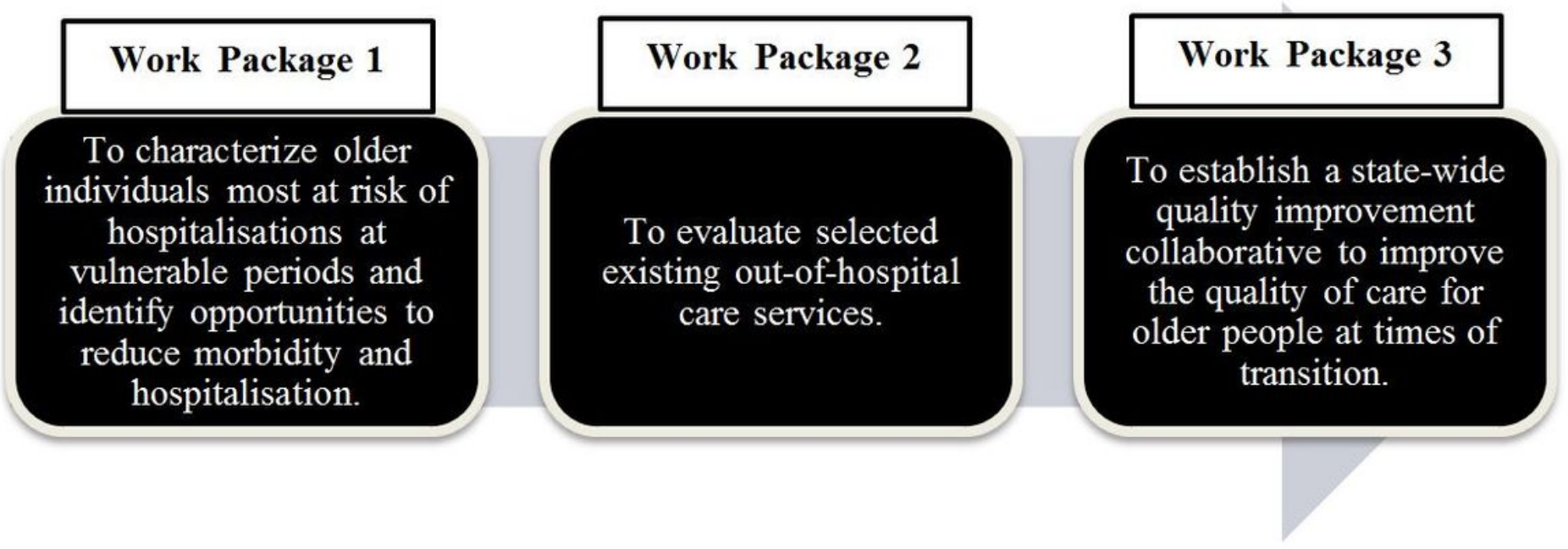

Figure 1

Overview of STAAR-SA Work Packages addressing aims 